Cahiers québécois de démographie

\title{
Familles montréalaises du XIXe siècle : trois cultures, trois
} trajectoires

\section{TRAJECTORIES OF THREE COMMUNITIES IN NINETEENTH-CENTURY MONTREAL FAMILIAS DEL SIGLO XIX EN MONTREAL : TRES CULTURAS, TRES TRAYECTORIAS}

\section{Sherry Olson et Patricia Thornton}

Volume 21, numéro 2, automne 1992

Montréal, $\mathrm{XIX}^{\mathrm{e}}-\mathrm{XX}^{\mathrm{e}}$ siècles : croissance urbaine et diversité culturelle

URI : https://id.erudit.org/iderudit/010121ar

DOI : https://doi.org/10.7202/010121ar

Aller au sommaire du numéro

Éditeur(s)

Association des démographes du Québec

ISSN

0380-1721 (imprimé)

1705-1495 (numérique)

Découvrir la revue

Citer cet article

Olson, S. \& Thornton, P. (1992). Familles montréalaises du XIXe siècle : trois cultures, trois trajectoires. Cahiers québécois de démographie, 21(2), 51-75. https://doi.org/10.7202/010121ar
Résumé de l'article

Afin de retracer l'évolution de la population montréalaise de 1840 à la fin du siècle, les auteures ont recueilli toutes les données se rapportant à douze patronymes dans les registres d'état civil (baptêmes, mariages et décès) et les recensements nominatifs. L'échantillon a été conçu de manière à permettre d'établir les mariages et filiations et de reconstituer intégralement quelques centaines de familles représentatives des trois principales cultures en présence : les Canadiens français, les anglo-protestants et les Irlandais catholiques. Chaque groupe a une structure par âge, des trajectoires de vie et des comportements démographiques spécifiques. 
Cahiers québécois de démographie

Vol. 21, no 2, automne 1992, p. 51-75.

\title{
Familles montréalaises du XIXe siècle : trois cultures, trois trajectoires
}

\author{
Sherry OLSON et Patricia THORNTON *
}

Nous tentons, à partir d'échantillons très petits, de tracer un portrait de l'évolution de la population montréalaise durant la deuxième moitié du XIXe siècle et de soulever quelques questions qui restent à approfondir. En cinquante ans, à la faveur de poussées de la construction et de vagues d'immigration marquées par les sommets de $1855,1864,1871$ et 1887 , la population urbaine décuple et sa structure par âge et sexe se transforme; les comportements démographiques paraissent se modifier aussi.

Pour dégager le sens de ces changements, il importe de départager les influences respectives des facteurs liés à la culture et des facteurs relevant de la classe sociale. Les études passées n'ont pas atteint cet objectif faute de données croisées (les recensements du XIXe siècle n'en fournissent pas) et de mesures valables du statut socio-économique; de plus, la quali-

\footnotetext{
- Respectivement du département de géographie de l'Universitê McGill et du département de géographie de l'Université Concordia. Remerciements : Outre le Conseil de recherches en sciences humaines du Canada, dont la subvention nous a permis de créer notre banque de données, nous tenons à remercier monseigneur Ivanhoë Poirier, p.s.s., curé, et le personnel du service des archives de la paroisse Notre-Dame, ainsi que monsieur Merle Christopher, du Cimetière Mont-Royal, de leur aimable collaboration. Nos remerciements vont aussi aux assistants de recherche qui ont consciencieusement recueilli les données sur lesquelles repose ce travail, et spécialement à André Duchesne. Merci également à Danielle Gauvreau, à Peter Gossage et à Jacques Légaré, qui nous ont fait bénéficier de leurs commentaires.
} 
té des registres disponibles pour la minorité anglo-protestante. qui détenait une grande part de la richesse, soulève des doutes. En 1847 , les 7500 ménages de Montréal composent une population mi-francophone mi-anglophone, comprenant environ un protestant et trois catholiques sur quatre. Les Irlandais, de même religion que la majorité canadienne-française et de même langue que la minorité protestante, conservent, avec la mémoire des famines qui les ont forcês à immigrer (le plus fort contingent est venu en 1847), une identité bien affirmée; ils ont encore, à la fin du siècle, des comportements distinctifs. En 1901, Montréal compte 65000 ménages, dont deux tiers de Canadiens français; un ménage sur cinq vit en banlieue, hors des limites de la ville. Les trois groupes sont fermement enracinés et ont chacun leur géographie résidentielle et leurs institutions sociales : ils forment trois sociétés.

\section{TROIS TRAJECTOIRES FAMILIALES}

L'histoire de trois couples permettra d'illustrer les possibilités et la complémentarité de nos sources. Tous étaient mariés en 1853 et les trois femmes ont porté leur dernier enfant à peu près au même âge, soit 44-45 ans. Leur seul trait particulier est d'avoir vécu plus longtemps que la plupart de leurs contemporains, de sorte qu'ils sont prêsents tout au long de la période qui nous intéresse. Longévitê mise à part, leur existence s'apparente à celle des membres de leur groupe.

François et Rose sont nés à Montréal et se sont mariés à 20 ans, comme beaucoup de couples canadiens-français. La figure 1 présente leur famille; on y distingue les données tirées de cinq recensements et les êvénements extraits des registres paroissiaux. Rose aura porté 15 enfants en 26 ans, dont un couple de jumeaux. Huit ont vécu seulement quelques mois, et le dernier-nê est mort à 5 ans, durant l'épidémie de variole de 1885. Le ménage comptera jusqu'à huit membres (au recensement de 1871), plus une servante. Les fils deviendront couvreurs, comme leur père; les petits-fils ajouteront la peinture et la plomberie. Au moins trois des enfants mariés sont morts avant Rose et François, que nous voyons une dernière fois en 1901, restés seuls avec leur petite-fille orpheline ${ }^{1}$.

1 François est mort en 1902, à 69 ans, Rose l'année suivante. 
Le ménage de François et Rose...

Recensement
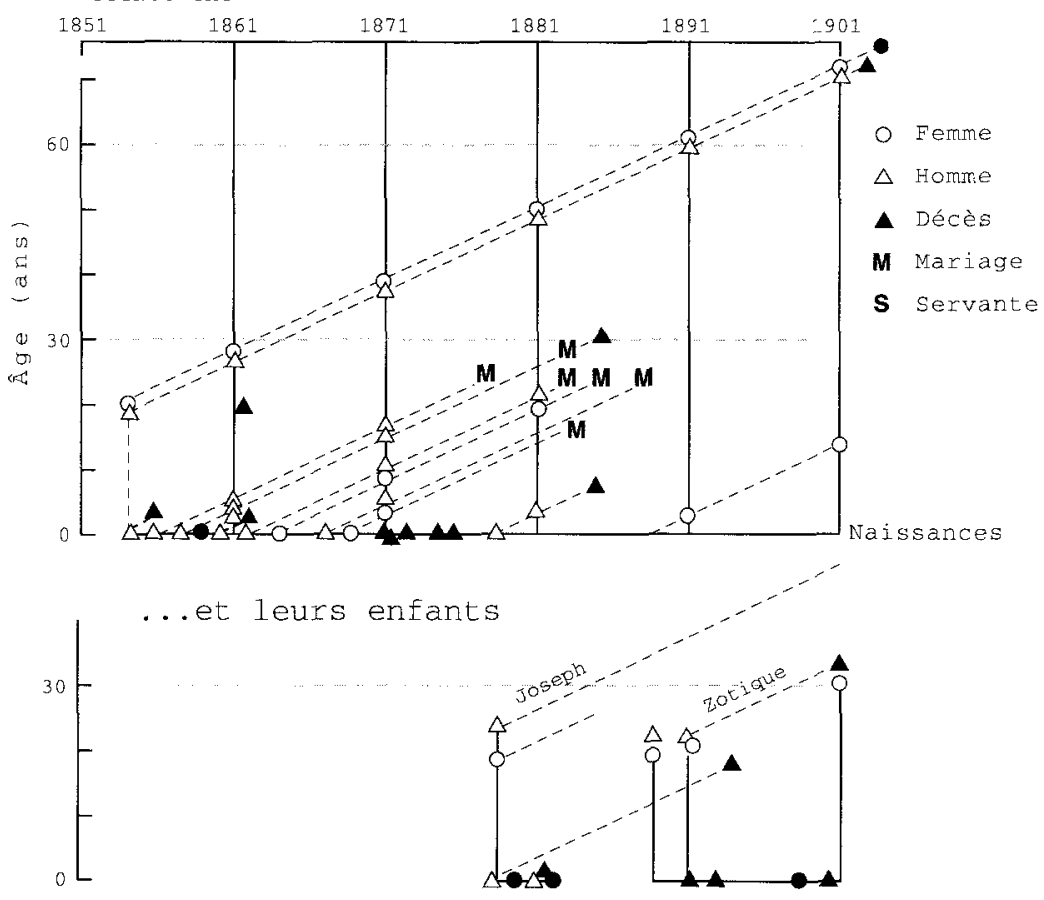

5

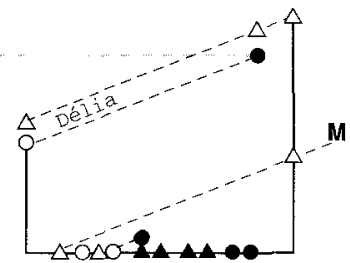

5

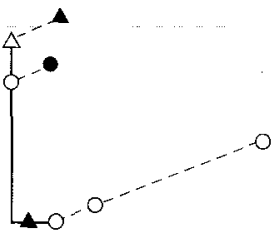

FIGURE 1 - HISTOIRE D'UNE FAMILLE CANADIENNE-FRANÇAISE 
À 24 ans, Harriet épouse William, 32 ans, issu d'une famille anglicane active dans la construction. Ils auront 12 enfants, qui franchiront tous la petite enfance; nous savons que huit se sont mariés et ont élevé des enfants à Montréal. Le jour de leur mariage, les filles de Harriet avaient entre 20 ans et 31 ans. Les fils sont restés dans le domaine de la construction, les uns comme artisans, les autres comme entrepreneurs. Le ménage a compté jusqu'à 14 membres, plus une servante à demeure. Malgrê trois naissances de moins que Rose (soit un cinquième), Harriet a élevé une fois et demie plus d'enfants, et pendant de nombreuses années son ménage a été près de deux fois plus grand (14 membres contre $8: 175 \%$ ).

Nés en Irlande, Mike et Maggie sont établis à Montréal lorsqu'ils se marient, lui à 30 ans, elle à 28 ; c'est leur première union à tous deux. Ils vivront au delà de la soixantaine. Mike est tisserand et "journalier" (manœuvre). Leurs sept enfants vont dépasser la petite enfance, mais seulement trois se marieront (les autres mourront à 6, 17, 20 et 32 ans). Maggie est donc, au jour du mariage, la doyenne de nos trois épousées. Son ménage aussi sera plus grand que celui de Rose, malgré l'arrivée plus tardive du premier enfant et les naissances plus espacées: l'intervalle intergénésique moyen est en effet de 26 mois pour Maggie, 24 pour Rose et 21 pour Harriet.

Les trois familles sont présentes dans les échantillons que nous avons tirés des recensements nominatifs de 1871,1881 , 1891 et 1901. Chacun des trois échantillons - un par groupe comprend 60 à 200 couples. Le tableau 1 présente notre base de données initiale. Pour la constituer, nous avons dépouillé les recensements manuscrits de 1861 à 1901 afin d'y trouver les ménages dont le chef portait l'un des douze patronymes choisis. D'autres personnes de même nom figuraient sur les listes; il s'agit habituellement de belles-mères ou de servantes ou pensionnaires célibataires ${ }^{2}$. Nous avons également passé en revue les registres de toutes les paroisses de Montréal (banlieues comprises) ainsi que le rôle municipal de la taxe locative pour $1848,1861,1881$ et 1901 , de même que l'annuaire Lovell pour toutes les années comprises entre 1848 et 1902 . La stratégie d'échantillonnage basée sur les noms de famille a été conçue pour permettre de tirer le meilleur parti possible des

2 Le nom de jeune fille des femmes mariées est rarement indiqué dans les recensements et souvent omis dans les registres des cimetières et paroisses protestants, mais figure généralement dans les registres des catholiques. 
TABLEAU 1 - Données initiales sur l'échantillon constitué à partir des douze noms de famille, par source, pour Montréal et la banlieue

\begin{tabular}{|c|c|c|c|c|c|}
\hline & & $\begin{array}{l}\text { Canadiens } \\
\text { français }\end{array}$ & $\begin{array}{l}\text { Irlandais } \\
\text { catholiques }\end{array}$ & Protestants ${ }^{a}$ & Total \\
\hline \multicolumn{6}{|c|}{ Registres paroissiaux ${ }^{\mathrm{b}}$} \\
\hline 1 & Baptêmes ${ }^{c}$ & 2478 & 1052 & 1111 & 4641 \\
\hline 2 & Décès & 1592 & 938 & 824 & 3354 \\
\hline 3 & Mariages, hommes & $663 \mathrm{~B}$ & 290 & 319 & $1272 \mathrm{~g}$ \\
\hline & Mariages, femmes & 656 & 277 & 293 & 1226 \\
\hline \multicolumn{6}{|c|}{ Autres sources } \\
\hline 5 & 1848, Rôle d'évaluation d & 8 & 14 & 21 & 43 \\
\hline 6 & 1848, Annuaire ${ }^{\mathrm{e}}$ & 16 & 17 & 28 & 61 \\
\hline 7 & 1861, Recensement $\mathrm{f}$ & 25 & 25 & 54 & 104 \\
\hline 8 & 1861, Rôle d'évaluation d & 35 & 51 & 52 & 138 \\
\hline 9 & 1861, Annuaire ${ }^{\mathrm{e}}$ & 47 & 53 & 65 & 165 \\
\hline 10 & 1871, Recensement ${ }^{f}$ & 94 & 62 & 54 & 210 \\
\hline 11 & 1881, Recensement $f$ & 112 & 76 & 66 & 254 \\
\hline 12 & 1881, Rôle d'évaluation & 94 & 64 & 91 & 249 \\
\hline 13 & 1881, Annuaire ${ }^{e}$ & 90 & 64 & 95 & 249 \\
\hline 14 & 1891, Recensement ${ }^{\mathrm{f}}$ & 92 & 62 & 48 & 202 \\
\hline 15 & 1901, Recensement ${ }^{f}$ & 178 & 81 & 88 & 347 \\
\hline 16 & 1901, Rôle d'êvaluation & 194 & 102 & 118 & 414 \\
\hline 17 & 1901, Annuaire ${ }^{c}$ & 198 & 102 & 157 & 457 \\
\hline
\end{tabular}

a. Pour les protestants, les chiffres comprennent quelques mariages et baptêmes de catholiques portant un patronyme "protestant"; nous avons exclu ces cas de l'analyse.

b. 1830-1920 (pour les Canadiens français, les données remontent plus haut).

c. Comprend des "ondoyés" et des isans baptéme* catholiques, de méme que les "mortnés" protestants.

d. Montréal seulement.

e. Annuaire Lovell (logements et places d'affaires).

f. Chefs de ménage portant lun des douze patronymes. Comprend aussi les veuves dont le nom de femme mariée fait partie de ces noms, mais non les pensionnaires, les domestiques et les chambreurs.

g. Comprend des cas de mariage célébré hors de Montréal (il s'agit de couples résidant à Montréal).

index et des répertoires généalogiques disponibles et de stratifier adéquatement les trois échantillons ${ }^{3}$.

3 Nous nous sommes inspirées de la stratégie exposée dans Bardet (1983), que nous avons appliquée aux cohortes de naissance décrites dans Thornton et Olson (1991; à paraître). Les petits èchantillons dont il est question ici sont stratifiés pour être représentatifs des trois sous-populations étudiēes; on ne peut donc additionner les chiffres pour obtenir des résultats globaux. Des comparaisons préliminaires avec les rôles de la taxe locative (échantillon à $100 \%$ ) et les cohortes de naissance (échantillon à $10 \%$ montrent que chacun des échantillons est représentatif au plan de la distribution des loyers, de la répartition géographique, de la mortalité infantile et de l'âge au mariage. 
On trouvera au tableau 2 des données sur la taille et la structure par âge des ménages des trois échantillons. Le ménage de l'époque est une unité économique qui dispense les services de buanderie, restaurant, pouponnière, garderie, infirmerie, hospice. Avoir un chez-sol, n'est-ce pas, suivant le mot cruel du poète, avoir un endroit "où l'on est obligé de vous accueillir quand vous devez y aller" 4 ... Si elles jouissent d'un revenu, les personnes seules peuvent toujours loger dans une maison de pension; sinon, il leur reste l'institution (voir par exemple Bradbury, 1992). Pour éviter ces solutions de dernier recours, des ménages inhabituels se constituent, tel celui-ci, de huit frères et sœurs orphelins dont aucun n'a encore vingt ans, ou cet autre composê de trois frères et de leur sœur (tous sont venus ensemble d'Irlande); on voit aussi un veuf, vivant sous le même toit que sa sœur célibataire, qui élève ses enfants, et un autre veuf, marié à la sœur de sa dêfunte femme.

Comme le montre la figure 2 , d'un recensement à l'autre, la population irlandaise et la population canadienne-française vieillissent. La tendance est moins nette chez les protestants, rajeunis par une immigration continue. Les familles canadiennes-françaises, établies dans l'île de Montréal au cours des années 1650, s'étaient ensuite, en l'espace de deux générations, rêpandues dans un réseau de villages de la plaine de Montréal. Durant les années 1850, on voit des individus et des couples regagner Montréal, et ce mouvement, stimulé par la croissance économique, reprend avec une intensité accrue au cours des années 1880 .

En 1901, les ménages qui n'ont pas d'enfant de moins de trois ans sont beaucoup plus nombreux que les autres. Le fait est particulièrement frappant chez les protestants dont le loyer est élevê et chez les Canadiens français (tableau 2). La procréation devient l'apanage de certains ménages, et une différenciation des stades du cycle de vie se précise.

Les femmes prédominent dans les trois échantillons: l'heure n'est plus à la conquête de nouvelles frontières, comme au XVIIe siècle. Le phénomène est particulièrement patent dans le groupe d'âge 15-25 (tableau 2), fait indicatif d'une importante redistribution des jeunes gens dans l'économie.

4 "Home is where when you have to go there they have to take you in*. Tire de The Death of the Hired Man* (Edward C. Lather, ed. Poetry of Robert Frost. Londres, Jonathan Cape, 1971: 71, traduction libre). 


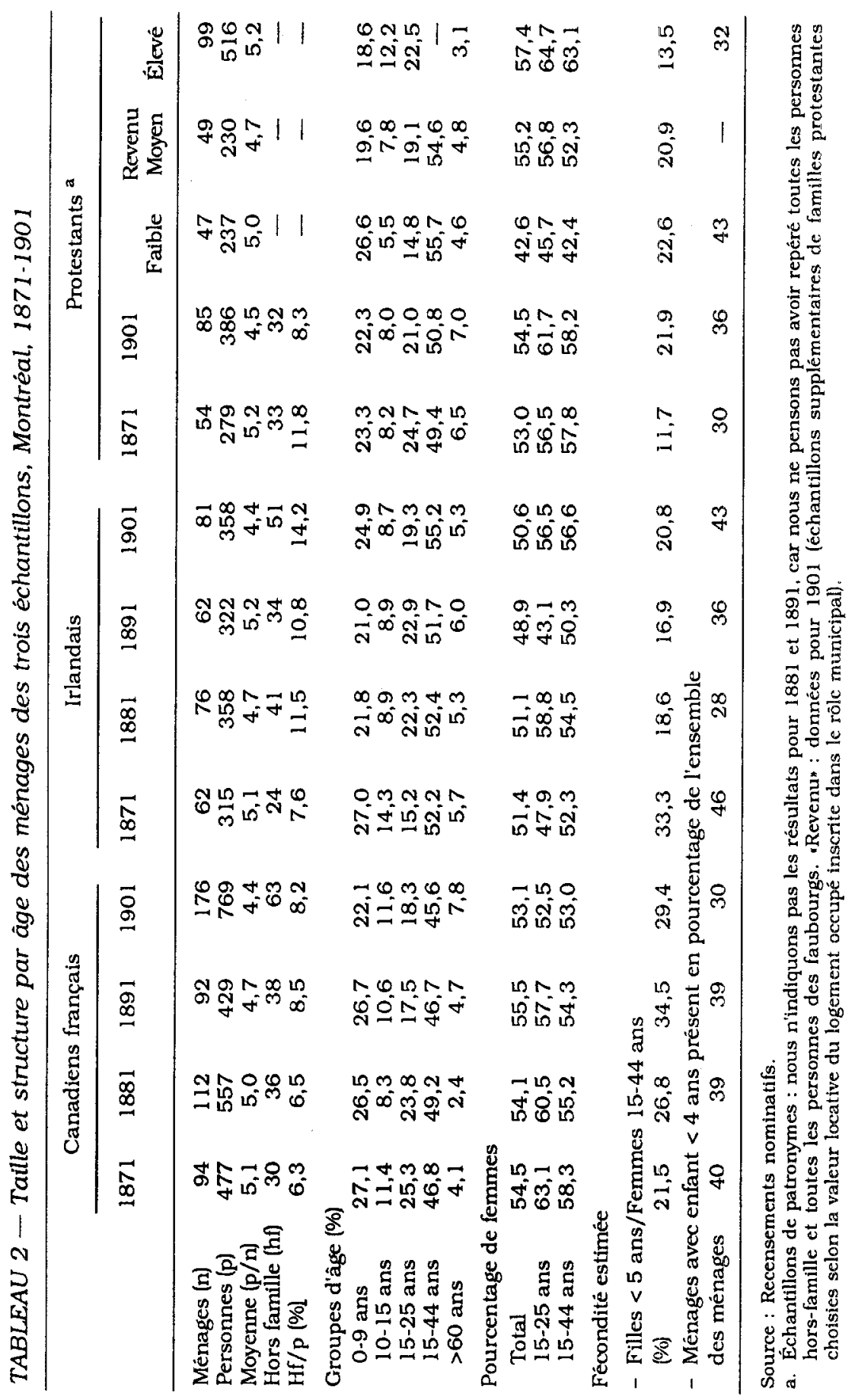




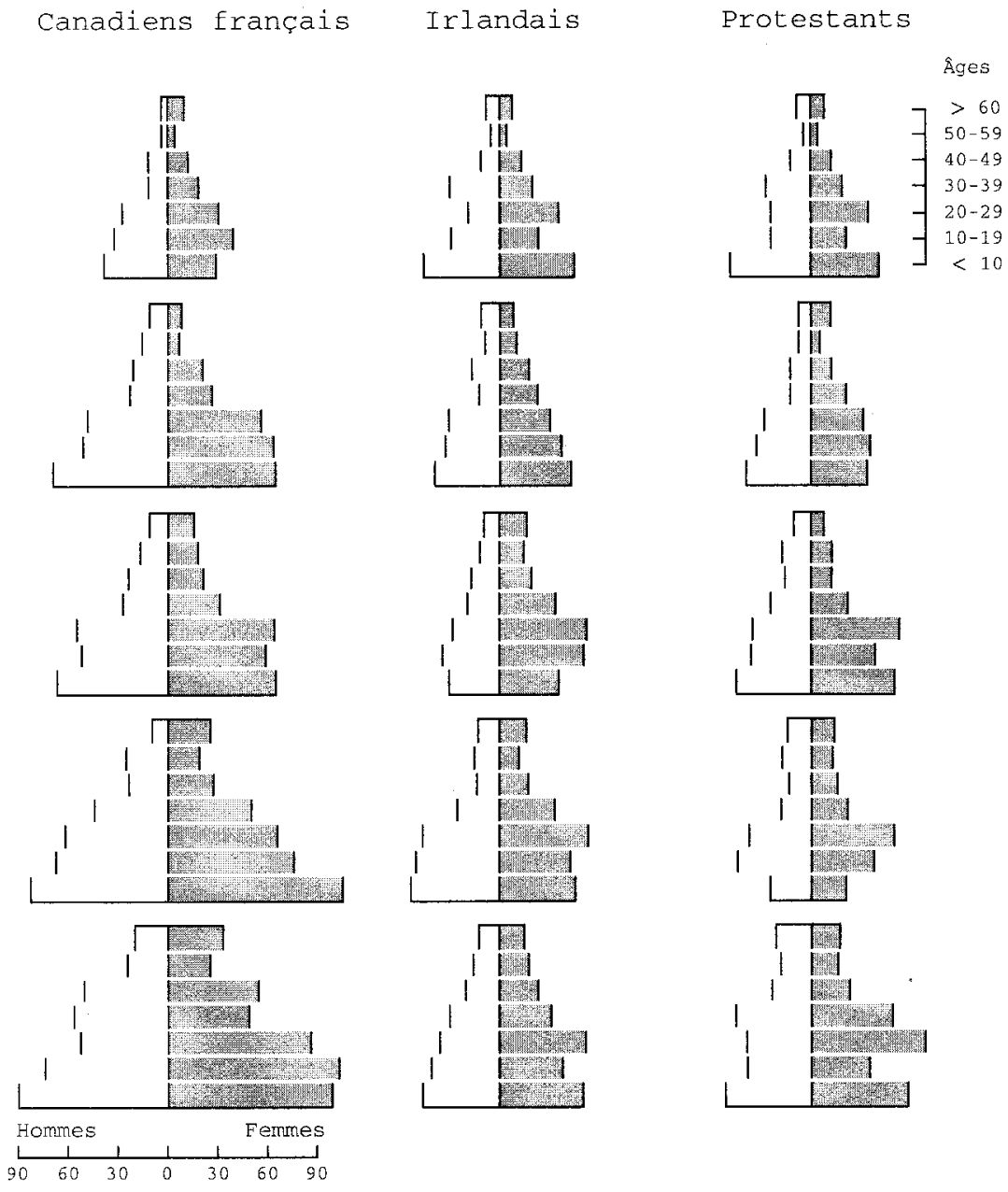

FIGURE 2 - STRUCTURE PAR ÂGE DES POPULATIONS DES TROIS ECHANTILLONS, 1861-1901

Deux tendances sont communes aux trois groupes : la réduction progressive de la taille moyenne des ménages (figure 3 ) et celle du nombre d'enfants présents. En 1861 et en 1871 , les enfants quittent la maison à 14-15 ans pour devenir domestiques, garçons de ferme ou apprentis et habiter chez leurs patrons. De la sorte, les jeunes hommes célibataires tendent à se disperser dans les campagnes environnantes tandis que les jeunes filles viennent en ville. La plupart des couples qui vivent à Montréal, y compris ceux dont les parents habitent un 


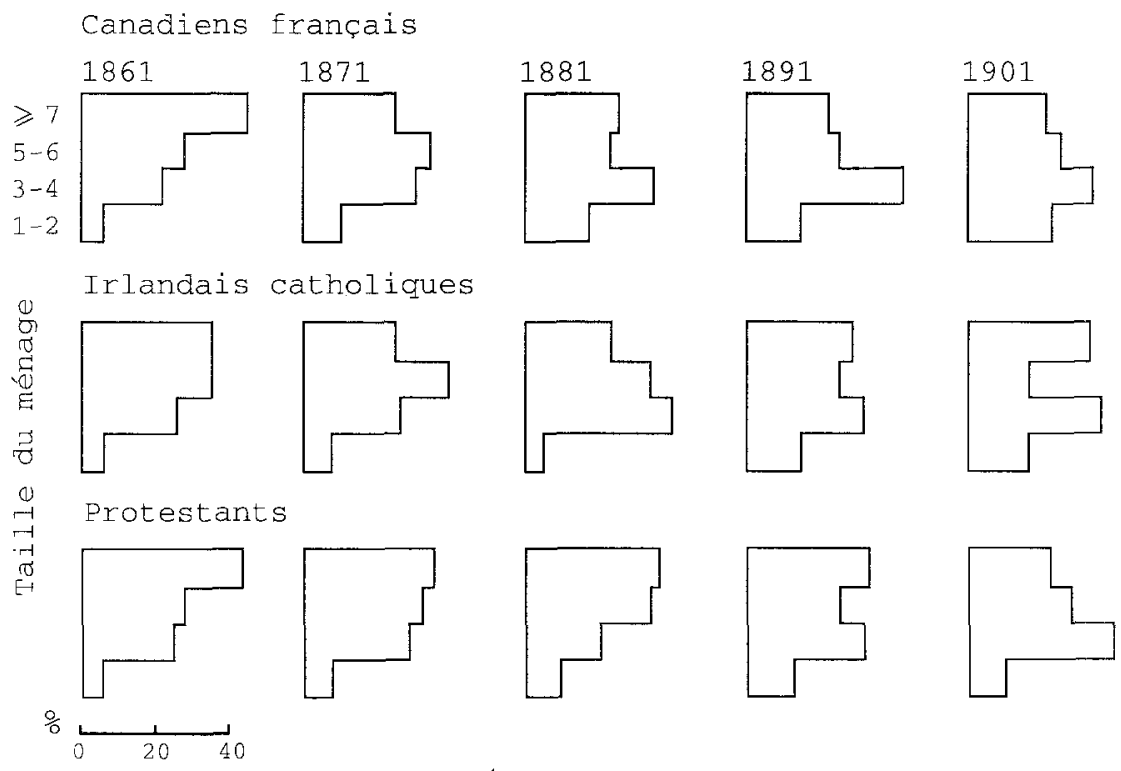

FIGURE 3 - TAILLE DES MENAGES DES TROIS ÉCHANTILLONS, 1861-1901

village, sont déjà installés dans une paroisse de Montréal au moment de leur mariage. À partir de 1891, et de façon plus nette en 1901 , de nouveaux types d'emplois s'offrent aux jeunes célibataires des deux sexes. Certains restent plus longtemps chez leurs parents, d'autres vivent en pension.

Ce sont les Irlandais qui présentent les transformations familiales les plus évidentes. Les premiers immigrés n'avaient pas à Montréal de parents à qui apporter leur soutien, mais leurs enfants, mariês au milieu des années 1860, doivent veiller sur leurs parents, veufs ou vieillissants. Déjà, en 1871 , la structure des ménages irlandais est particulièrement diversifiée et le groupe compte une proportion supérieure de personnes qui ne se marient pas, se marient tard ou ne se remarient pas en cas de veuvage. Vers la fin du siècle, le modèle de la vieille Irlande est rêaffirmê : un nombre à peu près égal de garçons et de filles restent célibataires et vivent chez leurs parents, souvent jusque dans la trentaine. À la fin du siècle, très peu d'Irlandaises catholiques se marient avant l'âge de 25 ans ou donnent naissance après l'âge de 40 ans.

La classe sociale exerce une influence sur ce modèle. En 1901, les domestiques sont moins susceptibles de vivre chez 
leurs maîtres qu'en 1861. Ils travaillent plutôt "de jour" et ne sont pas inscrits par les recenseurs comme membres du ménage de leur patron. Alors que, en 1861, les familles protestantes à l'aise avaient pour servantes des Irlandaises catholiques et des Canadiennes françaises, en 1901, elles cueillent les jeunes immigrantes protestantes à leur descente du bateau. Les femmes dans la trentaine prennent plutôt des adolescentes, les femmes dans la vingtaine des servantes de vingt ans ou plus.

Bien que très peu de domestiques habitent chez leurs maîtres en 1901, la taille des familles aisées dépasse la moyenne : comme en 1861, elles sont plus que les autres susceptibles de comprendre des neveux, des cousins, des frères ou sœurs, des tantes... Puisque les hommes les plus riches les $4 \%$ ou $5 \%$ qui occupent le sommet de l'échelle des revenus) se marient plus tardivement, les couples aisés continuent de présenter une plus grande différence d'âge entre conjoints.

La transformation de la structure des ménages s'accompagne d'une évolution de leur situation économique. Si, à l'échelle de la ville, la distribution des loyers, des revenus et de la quantité d'espace occupé bouge peu durant le demi-siècle sur lequel le rôle d'évaluation nous renseigne, nos échantillons témoignent de changements dans la position sociale relative des trois groupes (figure 4). En 1861, la communauté protestante est petite et de statut indéniablement élevé (on n'y trouve pas de journalier ${ }^{5}$ ), tandis que les chefs de ménage irlandais et canadiens-français sont concentrés dans les activitês à bas statut; ils vont toutefois enregistrer de nets progrès avant la fin du siècle ${ }^{6}$. Au dẻpart, les Irlandais sont les plus désavantagés : $44 \%$ sont journaliers, comme le quart des Canadiens français. En 1881 , les uns ont rejoint les autres (à quelque $25 \%$ ), et en 1901 la situation des Irlandais est relativement avantageuse. Le groupe protestant continue alors de monopoliser les revenus les plus élevés et ses familles bien établies peuvent s'enorgueillir d'exercer une gamme de professions étendue et d'alimenter l'expansion d'une classe de cadres. Mais on voit se former en son sein, parmi les jeunes hommes et femmes (1530 ans) qui ne vivent plus chez leurs parents, un segment de

51861 est l'année où nous commençons à suivre notre échantillon, qui est alors représentatif de toutes les catégories et de toutes les classes sociales. Les annuaires Lovell le confirment, mème si, bon an mal an, la proportion de journaliers y est sous-estimée d'au moins $10 \%$.

6 Les études sur la vie des Irlandais à Montréal sont peu nombreuses. Voir Cross, 1969, et Keep, 1948. 
Canadiens français
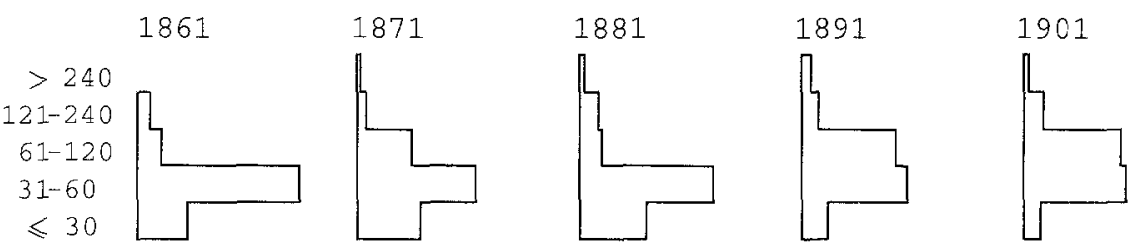

Irlandais catholiques
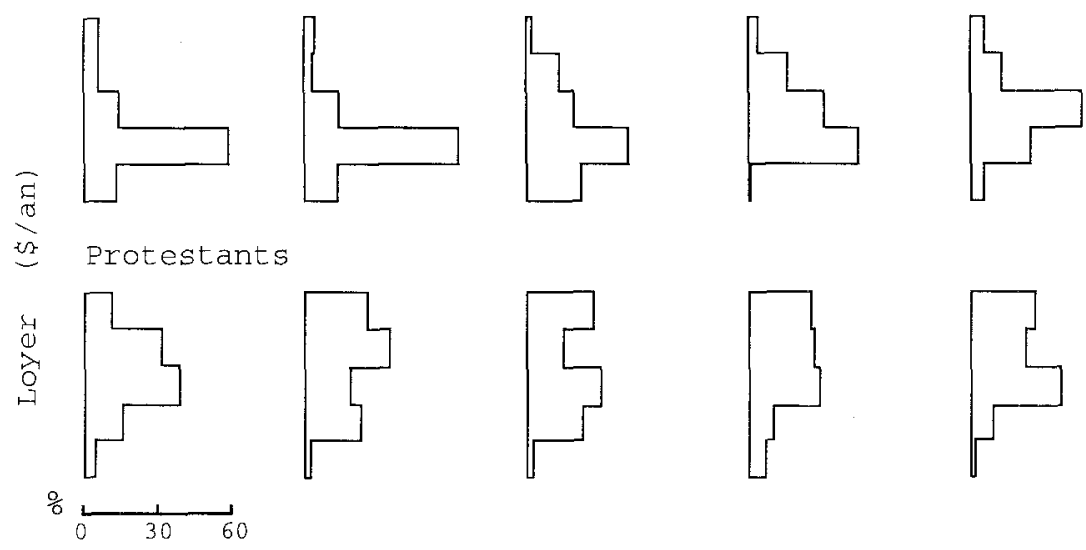

FIGURE 4 - DISTRIBUTION DES LOYERS DANS LES TROIS ECHANTILLONS, 1861, 1881 ET 1901

population qui paie des loyers peu élevés et fait face à une grande pauvreté.

La figure 5 représente l'évolution du nombre total de mariages, de naissances et de décès dans les trois communautés d'après les registres d'êtat civil; les données sont agrégées, et l'utilisation d'une échelle semi-logarithmique permet d'obtenir des courbes plus lisibles en mettant en évidence les variations des taux de croissance. La figure 6 (pour laquelle nous utilisons également une échelle logarithmique) représente l'évolution du nombre de ménages d'après l'information donnée par l'annuaire Lovell. Les Irlandais catholiques de notre êchantillon, après une période de croissance accélérée (pente fortement ascendante) atteignent assez vite un plateau ?

7 Ce résultat peut être influencé par le fait que l'êchantillonnage, reposant ici sur un seul patronyme, ne permet pas de tenir compte des familles qui viennent s'ajouter à la population irlandaise. L'échantillon canadien-français est également basé sur un seul nom de famille, tandis que l'échantillon protestant a été construit à partir de dix noms. 


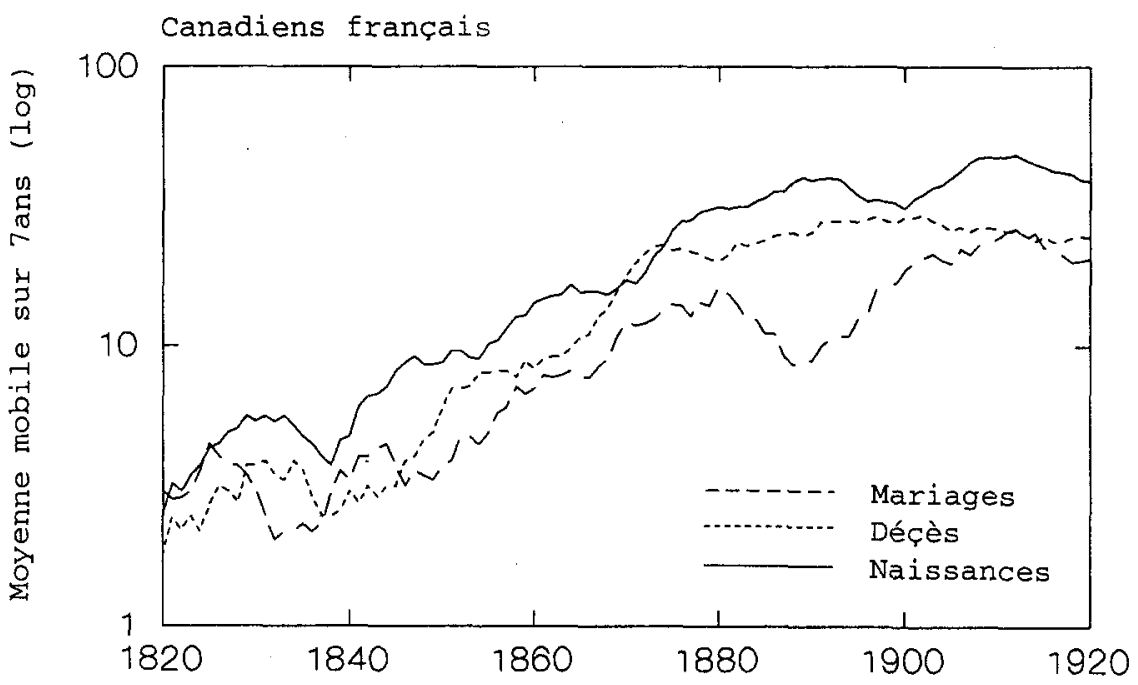

Source : registres d'état civil.

FIGURE 5 - MARIAGES, DECES ET NAISSANCES DANS LES TROIS ECHANTILLONS, 1820-1920 : CANADIENS FRANÇAIS

L'expansion de la population protestante met encore une décennie à ralentir. Quant au groupe canadien-français, il se stabilise plus tard encore et de façon plus graduelle. Sa courbe, dont le mouvement ascendant est, dans l'ensemble, le plus marqué, témoigne de sa croissance plus forte et plus soutenue tout au long du siècle.

Le rapport "naissances/ménages répertoriés" (figure 6a) pourrait, à première vue, laisser croire à un déclin de la fécondité. En fait les valeurs très élevées des années 1840 s'expliquent par la sous-représentation marquée des journaliers irlandais et canadiens-français dans les plus anciens annuaires. Cette carence reste sensible jusqu'à la fin du siècle (la sous-représentation est de l'ordre de $10 \%$ ) et accentue les diffêrences entre les communautés, mais la lêgère baisse qui se manifeste durant les années 1890 pourrait bien signaler une tendance au vieillissement et au report du mariage, sinon le début d'un réel déclin de la fécondité.

Vers 1880 , le rapport "naissances/mariages" des protestants tombe abruptement, et il reste ensuite beaucoup plus bas que celui des Irlandais catholiques (figure 7); ce résultat concorde avec les données des recensements sur la taille plus petite des familles protestantes. Mais les deux groupes anglophones sont largement distancés ici par les Canadiens 


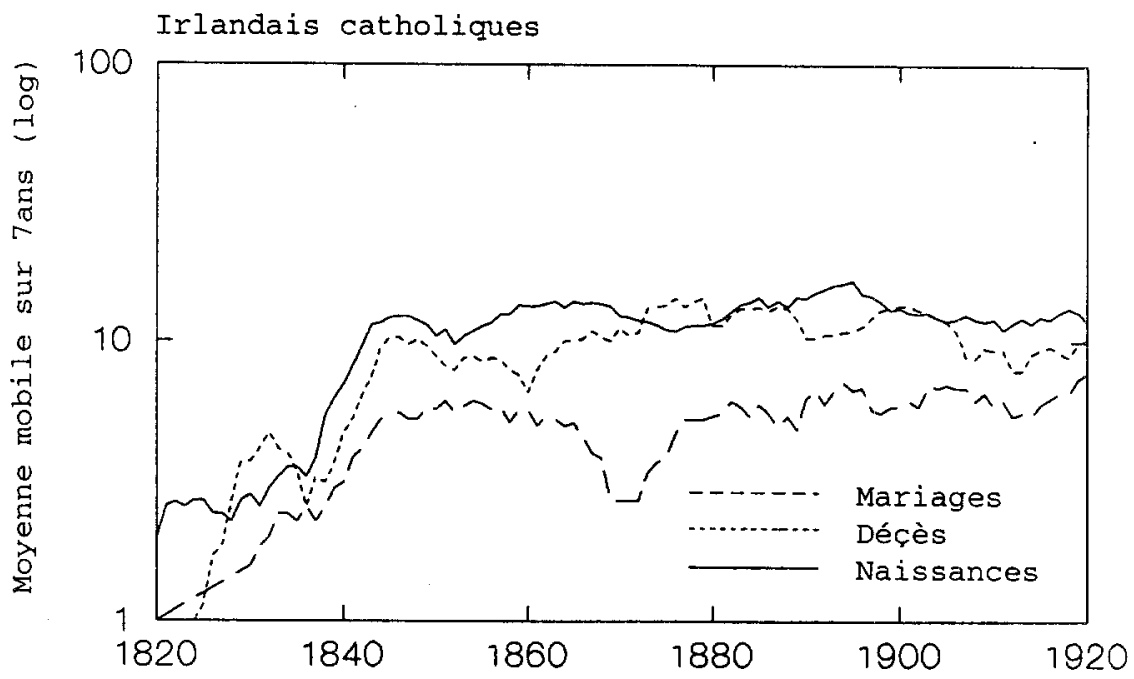

Source : registres d'état civil.

FIGURE 5 (SUITE) - MARIAGES, DECEES ET NAISSANCES

DANS LES TROIS ECHANTILLONS, 1820-1920 : IRLANDAIS

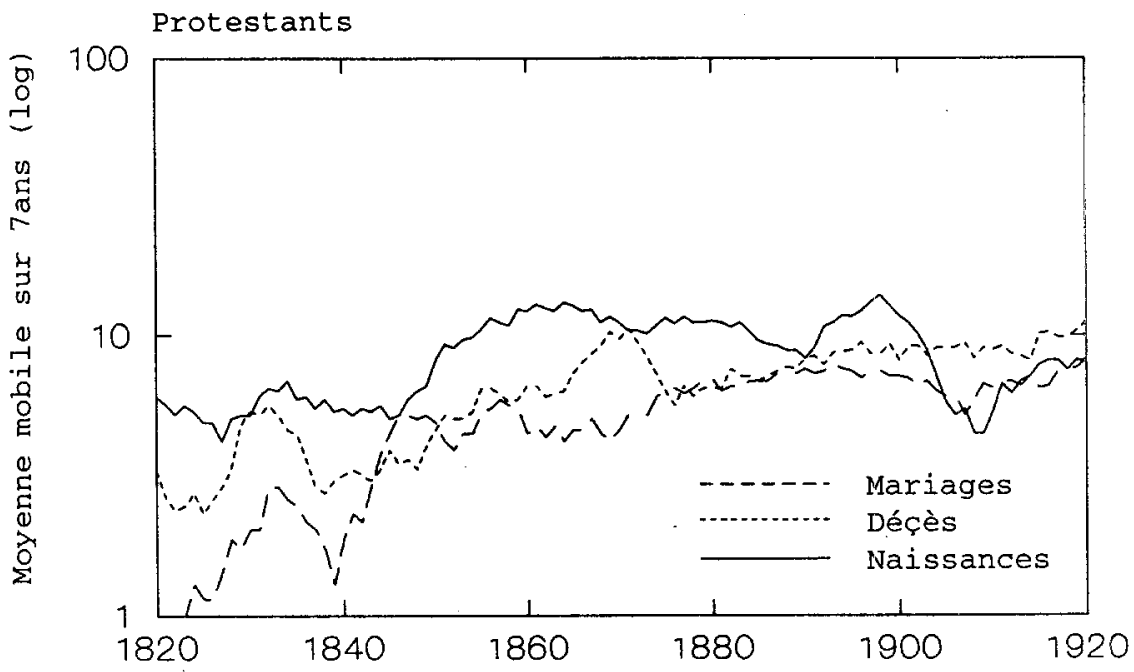

Source : registres d'état civil.

FIGURE 5 (SUITE) - MARIAGES, DECES ET NAISSANCES DANS LES TROIS ECHANTILLONS, $1820-1920$ : PROTESTANTS 
64

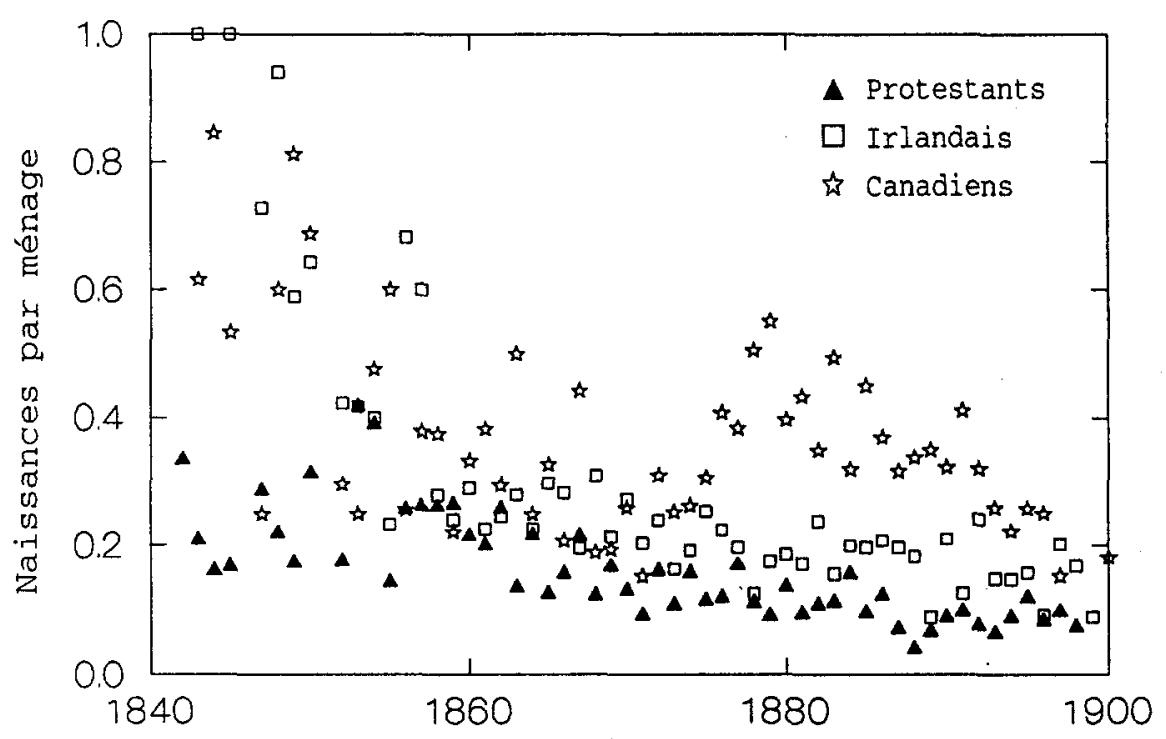

FIGURE 6A - NOMBRE MOYEN DE NAISSANCES PAR MENAGE

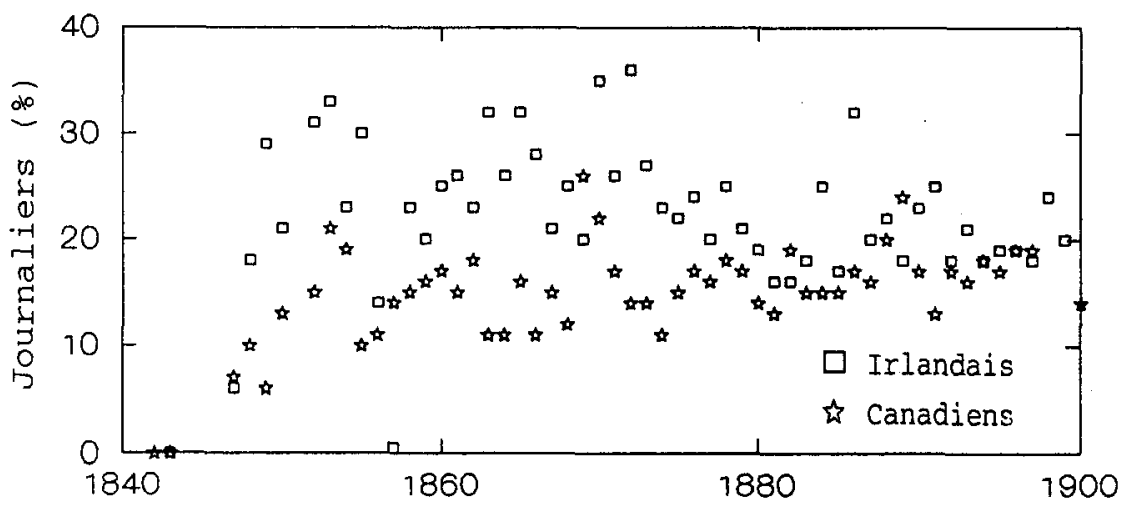

FIGURE 6B - POURCENTAGE DE JOURNALIERS PARMI LES CHEFS DE MÉNAGE 


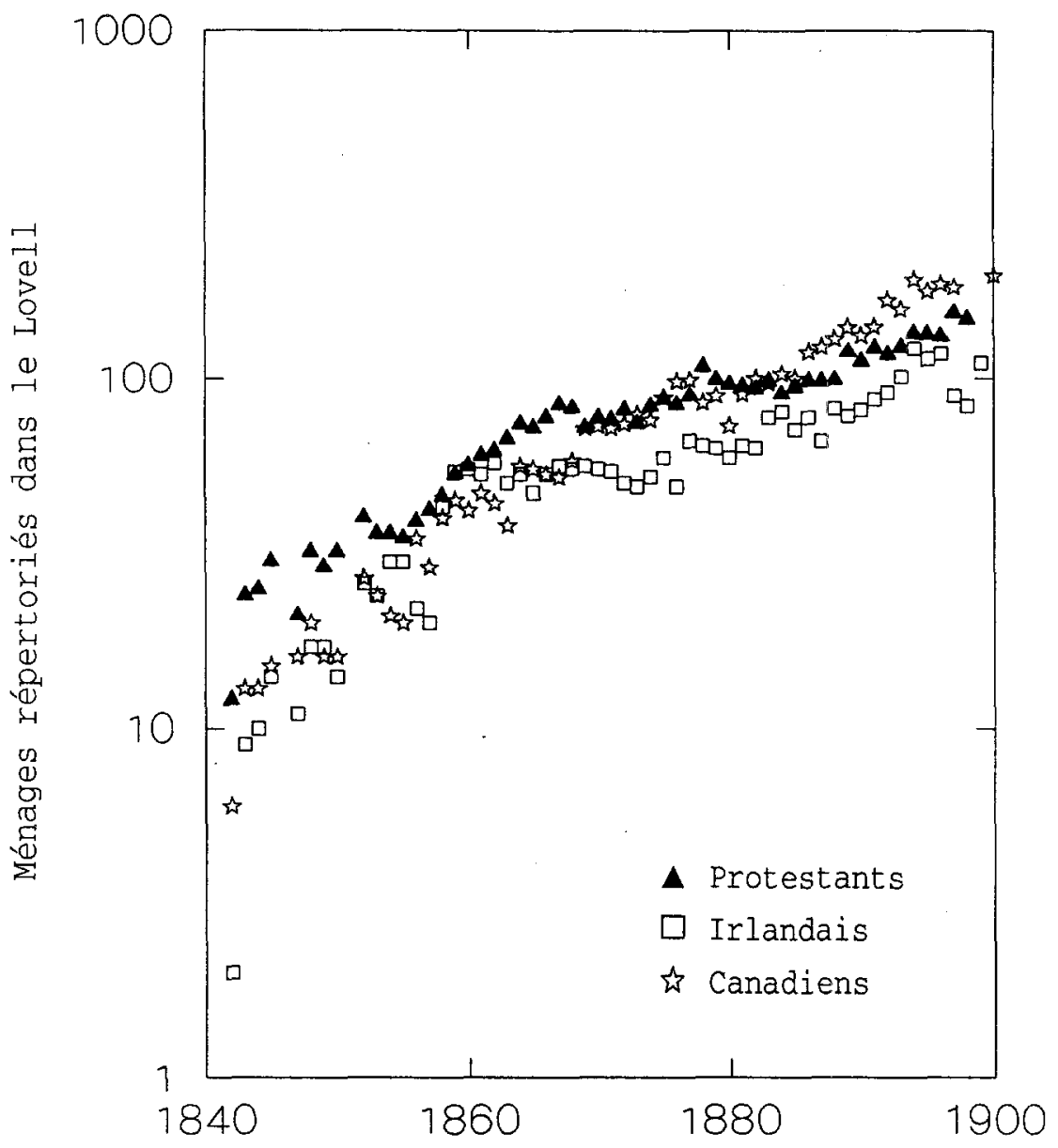

FIGURE $6 C$ - CROISSANCE NUMERIQUE DES MÉNAGES IÉCHELLE SEMILOGARITHMIQUE), POUR LES TROIS ÉCHANTILLONS, D'APRE'S LE LOVELL

français, au point que l'on peut parler de "revanche des berceaux" à l'échelle de Montréal.

Ces indicateurs soulèvent des questions de fond quant aux comportements et aux stratégies des ménages et des groupes. Comment concilier les familles nombreuses des Irlandais catholiques et la stagnation globale de leur effectif ? Comment conjuguer le déclin de fécondité des protestants et la jeunesse persistante de leur structure par âge ? Comment, enfin, la croissance des Canadiens français peut-elle se poursuivre alors que leurs ménages sont plus petits? 


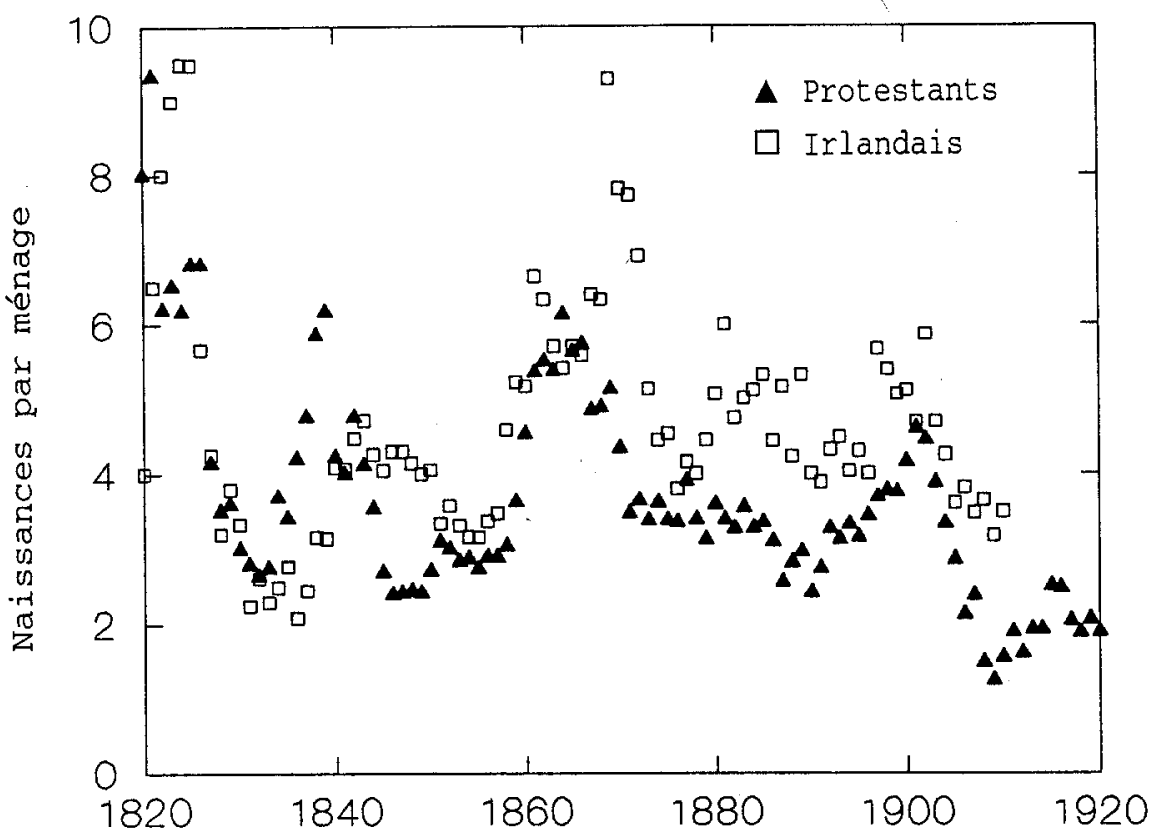

FIGURE 7 - RAPPORT DES NAISSANCES AU NOMBRE DE MARIAGES MASCULINS CHEZ LES IRLANDAIS ET CHEZ LES PROTESTANTS

\section{DIFFERENCES DE COMPORTEMENTS}

Pour répondre à ces questions, il faudra reconstituer les mille familles, vérifier les taux de migration (départs et arrivées) et évaluer la proportion d'actes manquants et de destins inconnus. En attendant, l'utilisation d'échantillons plus importants basés sur les cohortes de naissance ${ }^{8}$ permet de dégager quelques éléments d'explication. Ainsi, elle met en lumière de profondes différences entre les groupes eu égard à trois variables clês : l'âge au mariage, la mortalité infantile et l'espacement des naissances.

Si, à la fin du siècle, l'âge moyen au mariage tend à s'élever dans l'ensemble de l'échantillon (tableau 3), il n'en diffère pas

8 La question de la taille des échantillons est traitêe dans Thornton et Olson (1991; à paraître), où l'on trouvera aussi des renseignements méthodologiques et bibliographiques. Gossage, 1992, fournit des estimations de la mortalité des enfants en bas âge et de la fécondité des mariages dans la petite ville canadienne-française de Saint-Hyacinthe. 
TABLEAU 3 - Évolution de l'âge au premier mariage

\begin{tabular}{|c|c|c|c|c|c|c|}
\hline \multirow{2}{*}{$\begin{array}{l}\text { Date du } \\
\text { mariage }\end{array}$} & \multicolumn{3}{|c|}{ Hommes } & \multicolumn{3}{|c|}{ Femmes } \\
\hline & $\mathbf{N}$ & Moyenne & Mêdiane & $\mathbf{N}$ & Moyenne & Médiane \\
\hline
\end{tabular}

A. FAMILLES DE L'ECHANTILON a

Protestants

Avant 1880

$1880-1899$

28

28,9

26,1

25

24,1

23,3

$1900-1919$

25

$26,8 \quad 25,3$

41

$26,524,0$

26

$29,0 \quad 26,5$

31

$27,6 \quad 28,0$
B. ÉCHANTLLLON DE COHORTES : COUPLES AVEC JEUNE ENFANT (DONNEES DE RECENSEMENT) ${ }^{\mathrm{b}}$

Canadiens français

\begin{tabular}{llrlllll}
$1860-1879$ & 1881 & 601 & 23,8 & 22,0 & 633 & 20,3 & 20,0 \\
$1880-1899$ & 1901 & 954 & 25,0 & 23,7 & 948 & 22,1 & 21,2 \\
$\begin{array}{l}\text { Irlandais catholiques } \\
1860-1879\end{array}$ & 1881 & 108 & 25,9 & 24,0 & 105 & 21,5 & 21,0 \\
$1880-1899$ & 1901 & 58 & 26,9 & 26,2 & 63 & 23,8 & 23,4 \\
Protestants & & & & & & & \\
$1860-1879$ & 1881 & 142 & 25,3 & 24,0 & 124 & 21,0 & 20,0 \\
$1880-1899$ & 1901 & 36 & 26,6 & 26,0 & 34 & 24,1 & 23,1 \\
\hline
\end{tabular}

a. Les données sont basées sur les dates de naissance et de mariage des fils et filles des ménages de l'échantillon. telles qu'elles ont été recueillies dans les registres paroissiaux.

b. D'aprês les àges indiqués dans les recensements de 1861, 1881 et 1901 (la marge d'erreur est de 18 mois) et les dates de mariage notées dans les registres paroissiaux pour les parents d'un enfant inscrit dans le recensement: 1861, la totalité des ménages ayant un enfant de moins de 2 ans: 1881, enfants de moins de 3 ans (échantillon à $10 \%$ ): 1901 , enfants de moins de 4 ans (échantillon à $10 \%$ ).

Avant 1860 : la date du mariage manque pour une forte proportion d'immigrants, et le résultat pourrait en ètre faussé.

moins d'un groupe à l'autre. Ce sont les Canadiens français qui se marient le plus tôt, les Irlandais le plus tard. Parmi les femmes qui accouchent en 1879, par exemple, $59 \%$ des Canadiennes françaises se sont mariées avant leur majorité (avant d'avoir 21 ans), contre $52 \%$ des protestantes et seulement $42 \%$ des Irlandaises. Constat analogue pour les hommes: l'âge moyen au mariage est de deux ans plus bas chez les Canadiens français, et un sur quatre se marie avant d'avoir atteint la majorité. Chez les protestants qui forment le segment de population le plus riche (les $5 \%$ du haut de l'échelle), les hommes parviennent plus tard au mariage : un sur quatre 
attend d'avoir passé trente ans ${ }^{9}$. Cette tendance est significative : le report du mariage est un ajustement qui reflète des choix, des contraintes, l'apparition de nouvelles valeurs, et annonce peut-être d'autres changements.

Les taux de mortalité infantile des francophones présentent, par rapport à ceux des anglophones, des écarts très marqués qui vont persister jusqu'à la fin du siècle. Le quart des 4000 enfants nés à Montréal en 1859 n'atteignent pas leur premier anniversaire; la proportion est du tiers pour les Canadiens français ${ }^{10}$. Ces taux sont élevés par rapport à ceux de la plupart des villes britanniques et nord-américaines. Vers la fin du siècle, le taux commence à diminuer, mais plus lentement qu'ailleurs en Occident : $40 \%$ de l'ensemble des décès qui surviennent à Montréal continuent de toucher des enfants de moin d'un an, et près des deux tiers des enfants de moins de cinq ans ${ }^{11}$. Pour la cohorte de naissance de 1879 , le taux semble être $20 \%$ plus bas que pour celle de 1859 (tableau 4), mais cette baisse est le résultat momentané de variations annuelles très prononcées plutôt que l'expression d'une véritable tendance, et le fossé demeure : la mortalité infantile est $40 \%$ plus élevée chez les Canadiens français que chez les protestants et les Irlandais. D'après nos petits échantillons, l'écart persiste jusqu'à la fin du siècle. La mortalité infantile est de $29 \%$ dans la cohorte de naissance canadienne-française de 1899-1901, et la situation est peut-être en train de se détériorer chez les Irlandais.

Comment expliquer un tel écart ? Pour la cohorte de 1859 , la classe sociale n'exerce aucune influence évidente : les riches eux-mêmes ne parviennent pas à protéger leurs enfants. L'appartenance culturelle a un impact plus puissant que le loyer, la profession du père, la situation topographique ou la densité du bâti (Thornton, Olson et Thach, 1989). Pour la cohorte de 1879, l'impact du niveau de vie (mesuré par le loyer médian de la rue où habite la famille) est manifeste au sein des populations anglophones, dont la disposition géographique évoque le niveau social: les plus riches, êtablis à bonne hauteur (sur le flanc du mont Royal), ont délaissé les rues à forte densité et passent généralement l'été hors de la ville. Pour

9 Les proportions sont calculées par rapport au nombre de parents d'enfants nẻs en 1879-1880.

10 On compte $90 \%$ de décès chez les enfants trouvés ou abandonnés, pour la plupart nés hors mariage. Voir Gossage, 1987.

11 Rapports annuels du Département de santé de la cité de Montréal. 
TABLEAU 4 - Indices de mortalité infantile et de fécondité des mariages

A. MÉNAGES AYANT UN ENFANT DE MOINS DE 4 ANS, EN POURCENTAGE DE L'ENSEMBLE DES MÉNAGES, 1871-1901

\begin{tabular}{|c|c|c|c|c|c|c|c|}
\hline & \multicolumn{4}{|c|}{ CANADIENS FRANÇAIS } & \multicolumn{3}{|c|}{ IRLANDAIS } \\
\hline & \multicolumn{2}{|r|}{$\mathbf{N}$} & \multicolumn{2}{|c|}{$\%$} & $\mathbf{N}$ & & $\%$ \\
\hline 1871 & \multicolumn{2}{|r|}{95} & \multicolumn{2}{|c|}{40} & 59 & & 46 \\
\hline 1881 & \multicolumn{2}{|r|}{111} & \multicolumn{2}{|c|}{39} & 64 & & 28 \\
\hline 1891 & \multicolumn{2}{|r|}{93} & \multicolumn{2}{|c|}{39} & 59 & & 36 \\
\hline \multirow[t]{3}{*}{1901} & & 176 & 3 & & 86 & & 43 \\
\hline & \multicolumn{7}{|c|}{ PROTESTANTS } \\
\hline & $\mathrm{N}$ & $\%$ & $\mathrm{~N}$ & $\%$ & & $N$ & $\%$ \\
\hline 1871 & 54 & 30 & - & - & & - & - \\
\hline 1881 & 63 & 48 & - & - & & - & - \\
\hline 1891 & 56 & 30 & - & - & & - & - \\
\hline 1901 & 86 & 36 & 96 & 43 & & 99 & 32 \\
\hline
\end{tabular}

B. NOMBRE D'ENFANTS DE MOINS DE 4 ANS (ENF) DANS LA MÈME FAMILLE, 1901

\begin{tabular}{rrrrrrr} 
& \multicolumn{2}{c}{ CANADIENS FrançAIS } & \multicolumn{2}{c}{ IRLANDAIS } & \multicolumn{2}{c}{ ProtEstaNTS } \\
& $\mathrm{N}$ & $\%$ & $\mathrm{~N}$ & $\%$ & \multicolumn{1}{c}{$\mathrm{N}$} & \multicolumn{1}{c}{$\%$} \\
$\mathrm{ENF}=1$ & 1663 & 100,0 & 129 & 100,0 & 398 & 100,0 \\
$\mathrm{ENF}=2$ & 744 & 44,7 & 52 & 40,3 & 107 & 26,9 \\
$\mathrm{ENF}=3$ & 116 & 7,0 & 6 & 4,7 & 6 & 1,5 \\
\hline
\end{tabular}

C. NAISSANCES ET DÉCÈS INFANTILES POUR 1000 FEMMES MARIEES (TAUX ANNUEL BASÉS SUR LES NAISSANCES DE 1899 À 1901)

Can. Français Irlandais Protestants

Mortalité infantile (\%o) 289 273 97 Naissances pour 1000 femmes mariées, par an (moyenne sur 3 ans)

$$
\begin{aligned}
& 45-49 \text { ans } \\
& 40-44 \text { ans } \\
& 35-39 \text { ans } \\
& 30-34 \text { ans } \\
& 25-29 \text { ans } \\
& 20-24 \text { ans }
\end{aligned}
$$

Toutes les fermmes mariées

Taille de l'échantillon

Naissances à risque, mères $<20$ ou $>34$, pour 1000 naissances

$\begin{array}{rrr}0 & 0 & 0 \\ 82 & 100 & 100 \\ 183 & 167 & 143 \\ 314 & 154 & 212 \\ 403 & 318 & 240 \\ 385 & 250 & 400 \\ 230 & 164 & 189 \\ 123 & 45 & 55\end{array}$

Sources : A : Recensement de 1901, échantillons de patronymes, et échantillons supplémentaires de familles protestantes payant un loyer faible ou un loyer élevé. B : Recensement de 1901 , échantillon à $10 \%$ des familles ayant au moins un enfant survivant de moins de 4 ans. $C$ : registres paroissiaux pour les familles de l'échantillon de patronymes dans le recensement de 1901 . 
les Canadiens français, l'effet de la classe sociale est peu perceptible, même en 1879, mais les chances de survie des enfants sont d'autant plus grandes qu'ils habitent une rue moins densément peuplée et où les loyers sont en moyenne plus élevés. Ces caractéristiques du cadre de vie diminuent la probabilité qu'ils meurent pendant les mois d'êté ou succombent aux diarrhées (Olson et Thornton, à paraître).

Le caractère très saisonnier et la précision de ces causes nous conduisent à une explication plus complète et plus structurée. La moitié des décès d'enfants de moins d'un an se produisent toujours pendant les trois mêmes mois et ils correspondent de façon étroite à la surmortalité que les documents imputent à la diarrhée, au choléra des bébés et à la "dentition". Les maladies intestinales sont citées plus souvent pour les enfants canadiens-français. La suppression de la surmortalité de l'étê abaisserait la mortalité infantile du quart, et la combinaison observée à Montrêal - milieu urbain, hygiène déficiente et diarrhée - ressemble fort à celle qui a été remarquée en Grande-Bretagne au XIXe siècle (Woods et autres, 1988-1989) et plus anciennement dans le sud de l'Europe; elle rappelle aussi les ravages de la diarrhée chez les enfants après le sevrage en Afrique de nos jours. Les enfants qui ne sont pas nourris au sein sont plus vulnérables aux maladies imputables à la contamination de l'eau par les égouts. Nous savons aussi que la déshydratation peut être mortelle et qu'elle se combat par l'administration de liquides. Ces résultats nous amènent à rechercher les différences entre les cultures en ce qui concerne le soin des bébés, la durée de l'allaitement. les précautions entourant le sevrage durant la saison chaude et le soin des enfants malades.

Si nous nous sommes intéressées aux intervalles intergénésiques de chacun des trois groupes, c'est que nous cherchons à voir s'ils fournissent des indices de l'âge des enfants au sevrage. Dans la mesure où le système hormonal tend à freiner la fécondité de la mère qui allaite, exerçant ainsi un effet à la hausse sur l'intervalle intergénésique, la proportion d'intervalles inférieurs à 18 mois peut être un indicateur de la proportion de femmes "qui n'allaitent peut-être pas" (Nault et autres, 1990). En 1859, la proportion des mères qui semblent ne pas allaiter est similaire dans les trois groupes, mais en 1879 elle double chez les Canadiennes françaises (passant de $12 \%$ à $25 \%$ ), où elle atteint presque le double de celle des autres groupes. 
La mise au point de cet indicateur plutôt rudimentaire en vue de comprendre la mortalité infantile particulièrement élevée des Canadiens français nous a amenées à explorer d'autres aspects relatifs aux intervalles intergénésiques et à la fécondité. Dans la cohorte de 1859 , les intervalles médians des trois groupes sont proches du modèle huttérite, parfois présenté comme celui de la fécondité naturelle ${ }^{12}$. Pour la cohorte de 1879, nous avons, en augmentant et en stratifiant l'échantillon, fait ressortir certaines différences de distribution des intervalles entre les trois communautés. La rareté des intervalles courts (moins de 18 mois) caractêrise les mères irlandaises, tandis que chez les mères protestantes on note une plus grande variabilité des intervalles et un étalement des grossesses sur une plus longue période de vie. Les trois quarts des mères canadiennes-françaises présentent des intervalles concentrés autour de la médiane de 21 mois, donc courts par rapport à ceux des autres groupes, et de trois mois plus brefs qu'en 1860.

Ainsi les trois couples que nous avons décrits reproduisentils l'équilibre propre à leur groupe. Comme ses douze enfants ont survécu, Harriet s'est occupée d'une grande maisonnée, mais en 1901 elle n'avait pas beaucoup de petits-enfants, non plus que Maggie. Par contre, Rose, qui a perdu la moitié de ses enfants, n'a jamais eu auprès d'elle une famille aussi grande, mais en 1900 elle comptait à Montréal plus de descendants.

\section{GUELgUES CONJECTURES}

Les divers facteurs que nous avons évoqués sont interreliés, et chacun des trois groupes fonctionne suivant son équilibre propre. La Canadienne française se marie jeune, commence à procréer plus tôt que les autres femmes et finit plus tard, et ses enfants meurent en plus grand nombre. Un bébé étant mort, le suivant arrive plus vite et risque d'autant plus de subir le même sort. Les décès se succèdent parfois avec rapiditê: dans certains cas ils coïncident avec la mort de la mère ou du père. Dans notre cohorte de naissance de 1879 (plus de 1000 bébés), le temps moyen écoulê depuis le mariage des parents n'est que de sept ans, et presque tous les décès de parents de la période observée

12 Intervalle d'environ 24 mois lorsque l'enfant précédent a vécu plus de 12 mois, d'environ 18 mois lorsque l'enfant précédent est mort avant l'âge d'un an. 
sont dus à la tuberculose. Deux des enfants adultes de Mike et de Maggie succombent à cette maladie. Rose et François lui abandonnent aussi un fils, fauché avec sa femme après moins de cinq ans de mariage, et une de leurs filles meurt de "consomption" à 30 ans, peu après la naissance de son dixième enfant; la même semaine, le nouveau-né meurt "de faiblesse" et son aîné, qui a deux ans, de méningite tuberculeuse.

On peut explorer les raisons qui amènent les femmes à se marier plus âgées. Dans les strates supérieures, les hommes tendent à repousser le moment du mariage pour s'instruire davantage, s'initier aux affaires, voyager, s'établir, et le moment venu ils épousent des femmes plus jeunes qu'eux. Mais une différence d'âge de dix ans ou plus - très courante dans nos échantillons de protestants payant un loyer élevé - est synonyme de suprématie masculine dans le ménage et plus tard de veuvage prolongé. Ces deux raisons peuvent expliquer que les jeunes filles et leurs parents aient commencé à opter pour des mariages plus tardifs. En 1901, les loyers les plus élevés sont associès à des ménages plus grands, comprenant des adolescents et des jeunes, à forte prédominance féminine, qui renvoient l'image sans doute séduisante d'un stade de la vie consacré aux rencontres, aux loisirs et à l'apprentissage du monde.

À l'autre bout de l'échelle, le recensement de 1901 nous montre, plus nettement, une génération de jeunes qui sont forcés, souvent par mort de leur père ou de leur mère, de contribuer au revenu du ménage. Peu instruits, ils entrent dans la ronde des bas salaires: manufactures de soie, filatures de coton d'Hochelaga, usines de tabac ou de caoutchouc, couture à domicile. Parmi les jeunes hommes et femmes de 14 ans à 30 ans qui occupent ces emplois maigrement payés tout en vivant sous le toit familial, $44 \%$ des Irlandais et $33 \%$ des Canadiens français soutiennent un parent veuf. Tel est le nouveau prolétariat : une population d'adolescents obligés de se mettre à deux ou trois pour compenser la perte d'un adulte mieux payé parce que plus qualifié et plus expérimentê.

Dans les strates intermédiaires de revenu, on constate que l'économie apporte de nouvelles ouvertures : pour les femmes, des emplois de dactylo, de téléphoniste, de maitresse d'école, d'infirmière; chez les hommes, le nombre de voyageurs de commerce augmente. Déjà, en 1861 et en 1871 , les familles qui bénéficient d'un revenu un peu plus élevé ont tendance à garder leurs enfants sous leur toit plus longtemps. Elles le font par choix, et en 1901 les familles de la classe ouvrière agissent de 
même. Ces évolutions doivent être mises en rapport avec la tendance au report du mariage et avec le meilleur point de départ que représente, pour les jeunes couples irlandais et protestants, le fait d'avoir les moyens de se loger mieux durant leurs premières années de mariage.

\section{CONCLUSION}

Il appert que, malgré une mortalité infantile importante, la population de Montréal se renouvelle, contrairement à celle de Paris et d'autres villes européennes industrialisées de l'époque. Les conditions de vie sont toutefois plus favorables à la survie des enfants dans les villages, et la population rurale des environs de Montréal augmente plus rapidement. La croissance nette de la population canadienne-française de la ville s'explique à la fois par un solde migratoire positif dû à l'arrivée de jeunes célibataires et par l'accroissement naturel. Malgré la forte mortalité infantile, le groupe canadien-français s'accroit par rapport aux groupes irlandais et protestant; parmi les facteurs de son accroissement naturel élevé figurent le mariage plus précoce, le moindre espacement des naissances et le taux plus élevé de remariage des veufs et veuves.

On peut se demander si l'année du 350e anniversaire de la fondation de Montréal est le moment le mieux choisi pour évoquer la piètre qualité de l'hygiène publique et la mortalité infantile qui furent le lot de sa population. Rappelons toutefois qu'au début du siècle, comme les métropoles de bien des pays en voie de développement aujourd'hui, Montréal se caractérise par des infrastructures insuffisantes, une fécondité élevée et une grande diversité de modes de vie. La coexistence de cultures profondément enracinées accentue les variations locales des comportements démographiques, ainsi qu'on l'observe aujourd'hui à Dakar ou à Djakarta. L'hiver montréalais êtait en gênéral plus froid, plus enneigé et plus rude il y a un siècle, mais c'est en été que l'on comptait des morts en trop, sous l'effet de causes qui évoquent les conditions qui prévalent sous les tropiques.

Que Montréal ait été la capitale de la mortalité infantile, il y a lieu, non d'en rougir, mais d'admirer la ténacité de sa population. Si chaque personne peut avoir conscience du miracle que représente le simple fait de vivre, que dire de notre survie culturelle collective! Les registres paroissiaux, tenus par des générations de religieux, sont le produit et le témoin de la 
conservation de l'intégritê culturelle de Montréal. En tournant les pages, on est saisi par l'ampleur de ce patient miracle. Avant 1915, il n'existe au Nouveau Monde aucun moyen comparable d'observer les événements démographiques d'une ville en ayant sous les yeux l'éventail complet des différences entre cultures et classes sociales. Évidemment, Montréal est l'une des plus anciennes villes de l'Amérique du Nord. Au XIXe siècle, aucune autre ne réunissait autant de savoir et de culture et n'avait, pour la regarder vivre, des témoins aussi capables de conserver la trace des comportements de son peuple, tant savant qu'illettré.

\section{REFERENCES BIBLIOGRAPHIGUES}

BARDET, J.-P., 1983. Rouen aux XVI et XVIIIe siècles. Les mutations d'un espace social. Paris, SEDES, 2 vol.

BRADBURY. Bettina, 1992. "Mourir chrétiennement. La vie et la mort dans les établissements catholiques pour personnes âgées à Montrèal au XIXe siècle.. Revue d'histoire de l'Amérique française, 46, 1 : 143-175.

CROSS, S. 1969. The Irish in Montreal, 1867-1896. Montréal, Université McGill, mémoire de maitrise (histoire).

GOSSAGE, Peter, 1987. «Les enfants abandonnès à Montrêal au 19e siècle : la Crèche d'Youville des Sœurs grises, 1820-1871". Revue d'histoire de l'Amérique française, 40, 4 : 537-559.

GOSSAGE, Peter, 1991. "Family Formation and Age at Marriage in Saint-Hyacinthe, Quebec, 1854-1891". Histoire sociale/Social History, 24/47:61-84.

KEEP. G., 1948. The Irish Immigration to Montreal, 1847-1867. Montréal, Université McGill, mémoire de maitrise (histoire).

NAULT, François, Bertrand DESJARDINS et Jacques LEGARÉ, 1990. -Effects of Reproductive Behaviour on Infant Mortality of French Canadians during the Seventeenth and Eighteenth Centuries*. Population Studies, 44 : 273-285.

OLSON, Sherry, et Patricia A. THORNTON, à paraitre. "A Generation of Change in Montreal, 1860-1880n.

THORNTON, Patricia A., Sherry OLSON et Guoch Thuy THACH, 1988. "Dimensions sociales de la mortalité infantile à Montréal au milieu du XIXe siècle». Annales de démographie historique 1988 : 299-325.

THORNTON. Patricia A., et Sherry OLSON, 1991. «Family Contexts of Fertility and Infant Survival in Nineteenth-Century Montreal». Journal of Family History, 16, 4: 401-417.

WOODS, R. I., P. A. WATTERSON et J. H. WOODWARD, 1988-1989. The Causes of Rapid Infant Mortality Decline in England and Wales, 1861-1921", Part I : Population Studies, 42 (1988) : 342366: Part II : ibid., 43 (1989), $113-132$. 


\section{RÉSUMÉ - SUMMARY - RESUMEN}

OLSON Sherry et THORNTON Patricia - FAMILLES MONTREALAISES DU XIXE SIECLE : TROIS CULTURES, TROIS TRAJECTOIRES

Afin de retracer l'évolution de la population montréalaise de 1840 à la fin du siècle, les auteures ont recueilli toutes les données se rapportant à douze patronymes dans les registres d'état civil (baptêmes, mariages et décès) et les recensements nominatifs. L'échantillon a été conçu de manière à permettre d'établir les mariages et filiations et de reconstituer intégralement quelques centaines de familles représentatives des trois principales cultures en présence: les Canadiens français, les anglo-protestants et les Irlandais catholiques. Chaque groupe a une structure par âge, des trajectoires de vie et des comportements démographiques spécifiques.

OLSON Sherry and THORNTON Patricia - TRAJECTORIES OF THREE COMMUNITIES IN NINETEENTH-CENTURY MONTREAL

To trace the evolution of the population of Montreal from the 1840 s to the end of the century, we collected all baptisms, marriages, deaths and nominal census entries for 12 sample surnames. The sample is intended to permit matching. chaining, and a full reconstitution of several hundred families representative of each of the city's three principal cultural communities-French Canadian, protestant, and Irish catholic. The three communities functioned as distinctive subsystems, each with its own agestructure, vital rates, and behavioral patterns.

OLSON Sherry y THORNTON Patricia - FAMILIAS DEL SIGLO XIX EN MONTREAL: TRES CULTURAS, TRES TRAYECTORIAS

Para describir la evolución de la población de Montreal desde 1840 hasta fines de siglo, las autoras recolectaron todos los datos asociados a doce apellidos en los registros del estado civil (bautismos, casamientos $y$ defunciones), asi como los censos nominales. Se concibió el muestrario de manera a poder asentar casamientos y filiaciones, $y$ asi reconstituir integralmentevarios centenares de familias representativas de las tres comunidades culturales principales de Montreal: Canadienses franceses, angloprotestantes, e Irlandeses católicos. Cada una de estas comunidades tiene su propia estructura por edad, sus propias trayectorias de vida y sus propios comportamientos especificos. 